\title{
Beziehungsanalytische Traumdeutung
}

\author{
Andreas Hamburger (Berlin)
}

Zusammenfassung: Die Technik der psychoanalytischen Traumdeutung galt lange als eines der Urgesteine der Disziplin; sie schien die Paradigmenwechsel sowohl in Freuds Euvre wie in der späteren Psychoanalyse zu überdauern. Radikale Neuformulierungen wie die von Erikson, Morgenthaler und Meltzer wurden oft nicht oder nur oberflächlich angenommen. Obwohl sich die psychoanalytische Behandlungstechnik derzeit in Richtung eines beziehungsorientierten Ansatzes bewegt, scheint der Gestus des Deutens sich gerade im Bereich des Traums zu erhalten. Ausgehend von einer interpersonalen Entwicklungspsychologie des Traums untersucht der Beitrag das Geschehen in der psychoanalytischen Arbeit mit Träumen, mit einem Ausblick auf zeitgenössische Forschungsansätze zu non- und paraverbalen Aspekten des analytischen Dialogs.

Schlüsselwörter: Traumdeutung, Relationale Psychoanalyse, Beziehungsanalyse, psychoanalytische Behandlungstechnik

\section{Der erzählte Traum}

Der Traum, Freuds via regia zum Unbewussten, galt von jeher als Paradigma der inneren Welt. Wie kein anderes mentales und zugleich soziales Phänomen hat er das Interesse metapsychologischer Spekulation auf sich gezogen, hat Hirnforscher (Solms, 1997), Entwicklungspsychologen (vgl. Hamburger, 1987), Sozialund Literaturwissenschaftler beschäftigt (vgl. Alt, 2002). Einer der ersten klinischen Psychoanalytiker, die den (erzählten) Traum in seiner sozialen Dimension betrachteten, war Fritz Morgenthaler (1984, 1986, Binswanger, 2016). Sein Ansatz legt in nuce die Wurzeln einer Beziehungsanalyse des Traums (Hamburger, 1987, 1998a, 1998b, 1998c, 2000, 2006a, 2006b, 2010, 2013), die im Folgenden vorgestellt wird. Dazu sollen zuerst einige Begriffe geklärt werden; danach werden die entwicklungspsychoanalytischen und narratologischen Komponenten der Beziehungsanalyse des Traums erläutert und schliesslich am Beispiel eines Traumdialogs verdeutlicht. Den Abschluss bildet ein Ausblick auf die Funktion der Traumerzählung im Mikroprozess der therapeutischen Interaktion. 
Beziehungsanalyse: Relational oder interpersonal?

In der langen Geschichte der Psychoanalyse haben sich unterschiedliche Paradigmata abgelöst und überlappt. Die bestürzendste theoretische Volte schlug Freud selbst 1895. Im Entwurf einer Psychologie (Freud, 1950 [1895]) versuchte er «eine naturwissenschaftliche Psychologie zu liefern, das heisst psychische Vorgänge darzustellen als quantitativ bestimmbare Zustände aufzeigbarer materieller Teile und sie damit anschaulich und widerspruchsfrei zu machen» (ebd., S. 305). Dieser gross angelegte theoretische Wurf scheiterte noch während der Abfassung des Textes, Freud musste immer komplexere Modellannahmen konstruieren - und schliesslich gab er auf. Um zu erklären, wie im Reflexapparat ein Ich entstehen kann, musste er ein «hilfreiches Individuum» einführen (Freud, 1950, S. 365). Er meinte damit die Mutter, die das Schreien des Babys als Sprache (miss-)versteht und dadurch erst zur Sprache macht (vgl. Hamburger, 1987a, 3.2.3.3 und 1995, Kap. 2.1.2). Bereits an dieser frühen Stelle seiner Theoriebildung muss Freud eingestehen, dass soziale Interaktion die Voraussetzung der seelischen Entwicklung ist, dass Beziehung eingeschrieben ist in den «Apparat» (siehe dazu auch Derrida, 1967). Die klinische Konsequenz daraus zieht Freud freilich noch lange nicht. Sein Verständnis von Deutung folgt dem Modell der intrapsychischen Zensur; er sucht die bewussten Bereiche des Mentalen, also Gedanken, Wahrnehmungen und Introspektionen, insbesondere aber besondere mentale Phänomene wie Träume, Symptome und Fehlleistungen, durch Rekonstruktion unbewusster Zwischenschritte und ihrer Verbindung zu tabuisierten Wünschen erklärbar zu machen und dadurch neurotische, auf unbewussten Gleichsetzungen beruhende Symptome durch Bewusstmachung des kompletten Ablaufs zu neutralisieren. Dies wurde zunächst durch direktes Erraten der unbewussten Zwischenglieder und hypnotische Suggestion bewerkstelligt. Freud beobachtete dabei jedoch das unerwartete Phänomen der Übertragung (Freud \& Breuer, 1895, S. 308), und beschloss die Suggestionstechnik aufzugeben und an ihrer Stelle zur Technik der freien Assoziation überzugehen, unter Würdigung der Übertragungen als «Neuauflagen, Nachbildungen von den Regungen und Fantasien, die während des Vordringens der Analyse erweckt und bewusst gemacht werden sollen, mit einer für die Gattung charakteristischen Ersetzung einer früheren Person durch die Person des Arztes» (Freud, 1905, S. 179). Übertragung blieb dabei zunächst ebenfalls ein intrapsychisches Konzept. Als im Zuge der Spielrein-Affäre 1909 offenkundig wurde, dass diese Neuauflage nicht allein die Patientin erfasst, sondern auch den Arzt, konstatierte Freud defensiv, auch der Arzt [unterliege] einemähnlichen Prozess, der Gegenübertragung. Diese Gegenübertragung muss vom Arzt vollständig überwunden werden; das allein macht ihn psychoanalytisch mächtig. Das macht ihn zum vollkommen kühlen Objekt, um das der andere liebend sich bewerben muss. (Freud, in: Nunberg \& Federn, 1977, S. 407)

Mit der Ausrufung des «vollkommen kühlen Objekts» vermied er die naheliegende Konsequenz, die psychoanalytische Situation als eine gegenseitige zu betrachten. Ein Vorstoss wie Ferenczis Technik der mutuellen Analyse war wegweisend, aber für die Psychoanalytiker nicht beruhigend. Von Ferenczis Anstoss gingen zwei Entwicklungen aus: In der Tradition Melanie Klein-Heimann-Racker wurde Gegenübertragung als reaktiver, innerpsychischer Indikator der Beziehung aufgefasst, als Empfängerseite der projektiven Identifikationen des Patienten, während sie in der feldtheoretischen Psychoanalyse (Sullivan, vgl. Conci, 2005 2011) als ein Part im Kontinuum des interpersonellen Feldes galt. Beide Positionen waren im Mainstream zunächst schwer zu vermitteln. Die 1960er Jahre waren von umfänglichen Debatten um die technische und konzeptuelle Abgrenzung zwischen Analytiker und Analysand geprägt, die aus heutiger Sicht wie die Suche nach einem Archimedischen Punkt ausserhalb der Beziehung erscheinen (Hamburger, 1983).

Damit änderte sich auch die Deutungspraxis. In ihrem klassischen Verständnis als «technische» (Raguse, 1998) oder «rekonstruktive» Hermeneutik (Spence, 1976) hatte die klinische Psychoanalyse die Freilegung einer von unbewussten Verzerrungen entstellten «eigentlichen» Bedeutungsebene betrieben Paul Wachtel (1976) bezeichnet dies als «Wollhaarmammut-Hypothese», die davon ausgeht, dass die Wahrheit sozusagen im ewigen Eis der Verdrängung erhalten sei und nur aufgefunden werden müsse. Der interpersonale Ansatz dagegen verortet den analytischen Prozess nicht im Kontext der Archäologie, sondern in jenem der Detektivarbeit (Haubl \& Mertens, 1996). Nach Lorenzer (1985) begibt sich der Analytiker in einen Handlungszusammenhang mit dem Analysanden, so wie der klassische Detektiv sich in das «Outrierte» des Verbrechers einfühlt, spielt unbewusst mit in der sich entfaltenden Szene und reflektiert dies dann idealiter gemeinsam mit dem Patienten (Lorenzer, 1970). Der Analytiker achtet darauf, welche szenische Interaktion sich in der analytischen Situation entfaltet, welche Gefühle beim Patienten und beim Analytiker aufkommen. Er verbindet diese Figuren mit seinem Wissen um die Symptome und um die Lebensgeschichte des Patienten und lässt es zu, dass in dieser Verbindung neue, überraschende Zusammenhänge auftauchen. Überraschend sollten sie sein, sonst wären sie nicht 
der gesuchte unbewusste Sinn (Hamburger, 1998a). Deutung wird, nach dem Übergang von der einsichtsorientierten Ichpsychologie zur erlebensorientierten Selbst- und Objektbeziehungstheorie, nicht mehr als Subsumtion unter eine allgemeine Regel verstanden, sondern als Verbalisierung eines Beziehungserlebens. Deuten ist, mit einem Satz von Thea Bauriedl (1980, Kap. IV.3, S. 53 ff.), aussprechen "was in der Beziehung Bedeutung gewinnt".

Dieses Beziehungsparadigma findet sich in einer Reihe von zeitgenössischen psychoanalytischen Theorien und behandlungstechnischen Ansätzen, die unter Begriffen wie «interpersonal», «intersubjektiv» und «relational» bzw. im deutschen Sprachraum als «Beziehungsanalyse» firmieren. Leider ist die Begriffsverwendung oft schwankend und unscharf. Kunzke (2011) versucht eine Unterscheidung zwischen den aus der Sullivan-Schule stammenden interpersonalen Ansätzen, die sich wegen ihrer feldtheoretisch-systemischen Basis im psychoanalytischen Diskurs nicht wirklich durchsetzen konnten, den aus der Kohut-Schule bzw. der Objektbeziehungstheorie stammenden intersubjektiven Modellen, in denen die zentrale Bedeutung der empathischen Spiegelung bzw. der Integration von Beziehungsrepräsentanzen in der Entwicklung ebenso wie in der Therapie hervorgehoben wird, und schliesslich den vielgestaltigen relationalen Ansätzen, die seit Mitchell (1997) erheblich an Bedeutung gewonnen haben. Sie verbinden feldtheoretische Momente mit der selbstpsychologischen Forderung nach Empathie und Authentizität. Aufbauend auf den frühen interpersonalen Ansätzen von Harry Stack Sullivan und der Kulturalisten (Karen Horney und Erich Fromm) wurden sie seit den 1980er Jahren (Levenson, 1972, 1983, Greenberg \& Mitchell, 1983) einflussreich für den Mainstream-Diskurs, vor allem über die Zeitschrift Psychoanalytic Dialogues. Sie bilden zusammen mit sozialkonstruktivistischen Positionen eine neue Landschaft psychoanalytischen Denkens, die inzwischen als stiller Mainstream gelten kann. Eine wesentliche Schubkraft erhielt dieser Paradigmenwechsel von der Ein-Personen- zur Mehr-Personen-Psychologie aus dem feministischen Diskurs (Benjamin, 2009; vgl. Mertens, 2011).

Im vorliegenden Beitrag wird der Terminus «Beziehungsanalyse» gewählt, weil dieser frühe, international leider unbekannt gebliebene dialektische Ansatz (Bauriedl, 1980) die Arbeit des Verfassers geprägt hat, und weil sich aus seinem systemischen Verständnis des Unbewussten sowohl die Traumtheorie als auch die Techniktheorie der Traumdeutung am schlüssigsten entwickeln lassen. Von den genannten Ansätzen ist er am ehesten der interpersonalen Richtung zuzurechnen, vor allem in seiner Radikalität. Nimmt man als konstitutive Voraussetzung ernst dass weder Entwicklung noch Analyse in einem interaktionsfreien Raum denk- bar sind, so greifen intersubjektive und relationale Ansätze zu kurz. Sie anerkennen zwar die Bedeutung der Beziehung, doch analysieren sie weiterhin singuläre Psychen, und dies oft nach einem relativ festgelegten Muster. Kritisch merkt etwa Reimut Reiche (1999) an, das ...

(...) «Objekt» der Objektbeziehungstheorie [sei] immer ein «inneres Objekt», also das Movens eines durch inneren Zwang gesteuerten Beziehungsmusters. "Das Subjekt» als empirisches Wesen, als Individuum, als ganzer Mensch oder dergleichen war in der Psychoanalyse nie so sehr abgedunkelt wie im Postkleinianismus. Es soll gleichsam das transzendentale Subjekt aller möglichen empirischen inneren Objekte herausgearbeitet werden. (Reiche, 1999, S. 575)

Das «transzendentale Subjekt» ist hier eine höfliche Umschreibung de Tatsache, dass postkleinianische Deutungsmuster oftmals sehr gleichförmig ausfallen, als seien alle Patienten im Unbewussten mit dem binären Muster der guten vs. bösen Brust beschäftigt. Eine ähnliche Kritik könnte man an selbstpsychologischen Behandlungsberichten anbringen. Zwar ist die Kohut-Schule in der Tat mit der realen Präsenz des Anderen in Entwicklung und Analyse beschäftigt, doch liegt der Akzent weitgehend auf dem Aspekt der Wertschätzung. Die Fragmentierung, die bei deren Ausbleiben droht, wird inhaltlich weniger gefüllt als in der Objektbeziehungstheorie mit ihrer inneren Welt präambivalenter, noch im Modus der Spaltung befangener Subjekt- und Objektrepräsentanzen. So ist es in der Selbstpsychologie das Spiegelungsdefizit, das als Deutungsmuster immer wiederkehrt, um verschiedenste Pathologien zu erklären. Ganz ohne Zweifel sind Spaltung und Spiegelungsdefizite zentrale und klinisch höchst brauchbare Theoreme der klinischen Psychoanalyse. Es ist jedoch unwahrscheinlich, dass Menschen, die unter den unterschiedlichsten inneren und äusseren Bedingungen aufgewachsen sind, in der Struktur ihres Unbewussten so ähnlich sein sollen. Angesichts dieser Redundanz bedurfte es einer Theorie, die der Vielfalt klinischer Erfahrung besser entspricht. Die neueren relationalen Ansätze in den USA (Mitchell 1997, 2000, Orange, Atwood \& Stolorow, 1997) entstanden genau aus diesem Bedürfnis; sie verorten sich daher auch übergreifend zu metapsychologischen Paradigmata und unterstreichen nur die Dimension der Beziehung in der aktuellen analytischen Situation, die radikal als eine Zwei-Personen-Beziehung gesehen wird. Beide Beteiligten, Analysand und Analytiker, unterliegen unbewussten Prozessen, 
Abwehr, Verdrängung und Widerstand. Dass die relationalen Ansätze sich nicht auf ein Modell der Psyche festlegen, ist allerdings ein Problem, denn konzeptionell ist es ein grosser Unterschied, ob die emotionale Verfassung des Analytikers in der Sitzung als Inkarnation eines ideal empathischen mütterlichen Objekts, als Schauplatz projektiver Identifizierungen des Patienten oder als Teilhabe an einer unbewusst agierten Szene verstanden wird. Und es hat behandlungstechnische Konsequenzen. Interpersonale Ansätze wie die Beziehungsanalyse hingegen legen sich fest; sie gehen von der Dekonstruktion des Subjekts aus, betrachten das Unbewusste als eine systemische Entität, und akzeptieren damit einhergehend behandlungstechnisch den Übergang von der Deutungsmacht zum Dialog. Zur Frage steht nicht mehr, auf welchen okkulten Wegen das Unbewusste des Patienten in den Analytiker gelangt (Deutsch, 1926), sondern die systemische Verzahnung von unbewussten Beziehungsfantasien mit der Realbeziehung (Lorenzer, 1970b, Bauriedl, 1980). Die behandlungstechnischen Konsequenzen eines beziehungsanalytischen Ansatzes sind bedeutend, denn sie betrachten die Unbewusstheit des Patienten als gemeinsame Produktion. Das hat erhebliche Konsequenzen für das therapeutische Handeln des Psychoanalytikers. Wenn Unbewusstheit sozial erzeugt wird, auch in der analytischen Situation, so ist eine einsichtsvermittelnde oder deutende Haltung grundsätzlich zu hinterfragen. Sinnvoller scheint es dann, selbstanalytisch die Teilhabe an der aktuellen Produktion von Unbewusstheit (Erdheim, 1980) aufzudecken.

Nicht nur die Psychoanalyse, sondern das gesamte Wissenschaftsverständnis hat sich seither so grundlegend geändert, dass der Versuch, die spezifische Erfahrungsweise der Psychoanalyse begrifflich zu fassen anstatt sie einem physikalistischen Weltbild unterzuordnen, heute weit weniger exotisch anmutet als noch vor einem halben Jahrhundert, als solcheVersuche bereits vereinzelt unternommen wurden. Geht man nun also davon aus, dass der Geist keine Maschine, sondern ein in der Kommunikation gewachsenes und lebendes Ko-Konstrukt ist, so erfordert dies ganz neue Überlegungen zum epistemiologischen Status der Psychoanalyse. Axel Honneth hat seit 1992 eine Theorie der Anerkennung entwickelt, die mittlerweile ein Referenzmodell praktischer Philosophie geworden ist. Sie behandelt die konstitutive Rolle von Intersubjektivität: Jegliche innerpsychische Struktur ist Niederschlag einer primären Interaktionserfahrung, gibt dem Individuum aber auch eine gewisse Autonomie gegenüber der strukturierenden Umgebung (vgl. dazu Hamburger, 2016).
3 Traum ein Leben. Die interpersonale Konstitution der Innenwelt

Geht man vom Paradigma primärer Intersubjektivität aus, das auch die Säuglingsforschung gegenüber den Entwicklungsannahmen der früheren Psychoanalyse plausibel machen konnte (vgl. Dornes, 1993; Altmeyer, 2001), so ist auch der Traum keine Ausnahme. Tatsächlich lernen Kinder träumen. Die scheinbar selbstverständliche Unterscheidung der subjektiven Traum- von der intersubjektiven Wachwelt ist weder universal noch ist sie von Anfang an gegeben. Kleine Kinder erzählen Träume nicht anders als Erlebnisse aus dem Wachleben. Erst durch die Reaktionen der Erwachsenen lernen sie ihr Traumleben als solches vom Wachleben zu unterscheiden (vgl. Hamburger, 1987, 2006b). Wo und wie diese Unterscheidung gezogen wird, wie das Traumregister sozialisiert wird, ist eine kulturelle Option (Hamburger, 2013). Durch die Sinnzuschreibung der Eltern wird eine kulturell definierte Textsorte «Traum» generiert. In ihrem Eingehen auf die Traumerzählung «sortieren» sie für das Kind die innere (geträumte) Welt von der äusseren (wachen, intersubjektiven) und konstituieren dadurch erst im Erleben des Kindes die Traumwelt als eine innere, die nur dem Kind zugänglich ist.

Nach dieser ersten Phase der Traumsozialisation beginnen viele Kinde mit einem Mal, ihre Träume seltener zu erzählen, als wollten sie diese private Innenwelt schützen und für sich behalten. Erst später erzählen sie wieder öfter und man spürt dann einen gewissen Stolz. Aus dem kleinen Menschen, der so sehr in der Fantasie lebte, dass er Traum und Wirklichkeit fast gleich behandelt hat, ist ein Künstler geworden, der seine Schöpfungen präsentiert. Dieser Prozess ist eingebettet in einen unbewussten Traumdialog zwischen Eltern und Kindern. Nicht nur das Kind lernt träumen, auch die Eltern antworten durch ihre Einfühlung in die Traumproduktion des Kindes aus einer eigenen, im Hintergrund aktiven Traum-Matrix heraus. Sie ist verbunden mit ihrer unbewussten Beziehungsfantasie als Paar und mit dem phantasmatischen Kind (Brazelton \& Cramer, 1989). Durch die Partnerwahl entsteht ein unbewusstes Beziehungsfeld, in das Kinder hineingeboren und in dem sie sozialisiert werden. Die Sinnzuschreibungen in diesem Feld sind entwicklungsnotwendig und konstitutiv für die Entwicklung von Lebewesen, deren Gehirn darauf ausgelegt ist, Sinnzuschreibungen zu internalisieren. Die gegenseitige Beeinflussung findet nicht nur im Register des Traums statt, sondern auf allen Registern des affektiven und kognitiven Austausches. Strukturierte Verhaltenssequenzen des Kindes werden mit einer die Struktur aufgreifenden und zugleich transzendierenden Handlung in enger zeitlicher Abstimmung beantwortet. Indem wir sie bejahen und akzentuierend ausgestalten, nehmen wir Teil an jenem Ballett, das die Entwicklung der Beziehung und in deren Rahmen auch 
die psychische Entwicklung trägt. Wenn Eltern also angesichts ihres im Schlaf lächelnden und nuckelnden, wenige Tage alten Säuglings glauben, dass er vom Stillen träumt (Hamburger, 1999), so haben sie damit wahrscheinlich (noch) keine zutreffende Beschreibung seiner innerpsychischen Prozesse gegeben, aber sie bereiten ihn darauf vor, ein Träumer zu werden.

\section{$4 \quad$ Der Traum als Erzählung}

Die soziale Übermittlung von Träumen geschieht in aller Regeln in Form einer Erzählung. Auch dies ist keine Äusserlichkeit, sondern gehört, wie wir gesehen haben, zu den konstitutiven Elementen des Traums. Erst der erzählte Traum wird ja als solcher offenbar; solange er unerzählt im subjektiven Erleben bleibt, ist er (jedenfalls dem Kind) mit derWirklichkeit gleichwertig. Erzählen ist eines der zentralen Organisationsprinzipien der kindlichen Entwicklung. Stern (1989) beschreibt als Motiv des kindlichen Erzählens eine Störung ("The engine of the narrative is trouble”), als Ziel aber die Entwicklung von Kohärenz und Kontinuität und damit eines narrativen Selbst (Stern, 1985). Die auf der Stufe des verbalen Selbst erworbene persönliche Welterfahrung, die Erfahrung verbalisierbarer Urheberschaft und Geschichtlichkeit werden nun in ein narratives Selbst überführt. Erst damit entsteht eine rekonstruierbare Vergangenheit (Stern, 1989, S. 319). Ähnlich kann die Entwicklung des Träumens verstanden werden: Um mit neuen Erfahrungen umzugehen und sie ins Langzeitgedächtnis zu integrieren, simuliert der Träumer in wiederholten Entwürfen, in Form einer inneren Erzählung, mögliche Welten, die zu der unintegrierten Erfahrung passen könnten (Moser \& Zeppelin, 1996). Die Traumerzählung in der dyadischen analytischen Situation verbindet zwei lebendige Traumprozesse im Hier und Jetzt. Deshalb wird in den beziehungsorientierten Ansätzen der modernen Psychoanalyse (verschiedener Provenienz) die Erzählsituation genau beachtet (z. B. Aron, 1989; Ávila \& Domínguez, 2014; Caligor 1996; Fosshage, 2013a, 2013b). Nicht alle freilich folgen dem radikalen Gedanken dass die Abgrenzung von Traum und Realität erst in der Interaktion konstituiert wird und dass die Traumerzählung eine Neuauflage dieser wirklichkeitskonstitutiven Begegnung ist.

Diesen radikalen Ansatz aufzugreifen, war - wenn auch auf sehr verschiedene Weise - das Verdienst von Fritz Morgenthaler und Donald Meltzer Morgenthaler (1984) geht davon aus, dass Träume schon in ihrer Produktion auf den Analytiker bezogen sind, der sie hören wird: «Wenn mir ein Patient einen Traum erzählt, frage ich mich immer sofort, warum erzählt er mir diesen Traum jetzt, welche Botschaft an mich persönlich enthält dieser Traum.» Diese klinische
Haltung folgt aus seiner Annahme, dass die «Es-Anteile» des Traums nicht im Inhalt und auch nicht in den Assoziationen, sondern in der Mitteilungssituation zu finden seien, in der Stimmung, in welcher erzählt wird und im Umgang mit dem Traum.

So wie sich aus der Summe der Übertragungsäusserungen im analytischen Prozess gleichsam pantomimisch das anachronistische Erlebnismuster abzeichnet, widerspiegelt sich die unbewusste Traumtendenz an der Summe der Umgangsweisen des Träumers mit seinem Traum. (Morgenthaler, 1984, S. 69

Der Traum erscheint hier nicht als eine erst zu versprachlichende psychische Entität, sondern als ein Akt der Kommunikation, eingebettet in komplexe unbewusste Wahrnehmungs- und Delegationsprozesse. Das durch die Traumdiagnostik abgesteckte Feld erlaubt es erst, die Trauminhalte richtig zu lokalisieren (ebd. S. 87); die «Traumdiagnostik» ist also kein Widerspruch, sondern eine notwendige Erweiterung der Freudschen Traumdeutung, nämlich ihre radikale Anwendung auf die analytische Situation (vgl. Morgenthaler 1986, S. 42 f.). Insofern wird das Modell der «autoerotischen» Selbstanalyse, das Freuds Technik der Traumanalyse auch an Patienten bestimmte, aufgehoben durch die interaktionelle analytische Situation (Binswanger, 1986). Was dem «autoerotischen» Verstehen angemessen war - die Beschränkung auf Assoziationen - wird im alloerotischen Zusammensein von Träumer und Analytiker zum Modus der Abwehr: Die Aufforderung an den Träumer «frei zu assoziieren", bedeutet den Verzicht des Analytikers auf die eigenen Reaktionen. Sie schränkt den Träumer auf den «autoerotischen» Modus der Deutung durch Assoziation ein, klammert das szenische Geschehen aus. «So erhält man nicht die weiterführenden Assoziationen, sondern Rationalisierungen» (R. Binswanger, 1986, S. 180).

Eine Traumerzählung in einer Analysestunde ist in erster Linie ein Geschenk des Analysanden an den Analytiker, ein Vorgang, der beiden emotional nahegeht. Der Analytiker muss in der Lage sein, sich emotional auf dieses Geschenk einzulassen und es gleichzeitig zulassen zu können, dass er zunächst gar nicht versteht, was da geschieht. (Morgenthaler, zit. nach Heinrichs, 1986, S. 13)

Ähnlich begibt sich im Modell von Lorenzer (1970) der Analytiker in das Übertragungsfeld und versucht, es durch ein inneres Hin- und Heroszillieren zwi- 
schen Teilhabe und Reflexion deutend aufzulösen. Dies aber immer nur schrittweise; so dass die Deutung niemals eine Mitteilung fertiger Erkenntnis ist, sondern jeweils nur die Benennung des Schrittes, der sich eben in der therapeutischen Beziehung ergibt - was wiederum an das beziehungsanalytische Diktum von Bauriedl (1980) erinnert: «Deutungen sind das, was in der Beziehung Bedeutung gewinnt.» Um diese Haltung einnehmen zu können, ist der Analytiker auf seine Innenwahrnehmung angewiesen, denn nur in den in ihm selbst aufsteigenden Fantasien, welche auch immer es sein mögen, kann er seine unbewusste Teilhabe an der Szene erkennen. Diese Autoren verstehen die Deutung der Szene in einem ideologiekritischen Sinn.

Einen ähnlich starken Akzent wie Fritz Morgenthaler legt Donald Meltzer (1984) auf die eigene Teilhabe am Traumgeschehen - wenn auch auf einer gänzlich anderen theoretischen Grundlage. Meltzer geht vom Containermodell seines Lehrers Wilfred Bion aus und sieht die Analyse analog zur Beziehung zwischen Mutter und Kind. Die Mutter nimmt die unverdaulichen Aspekte des kindlichen Seelenlebens in ihre eigene Reverie auf und reagiert aus dieser träumenden Einfühlung so, dass sich im Kind Traumgedanken bilden können. Analog dazu löst die Traumerzählung im Analytiker eine spontane und unbewusste Antwort aus. Donald Meltzer (1984) hält Träume für so extrem verständlich, dass ihre Mitteilung die Peinlichkeit einer ungewollten Offenbarung bewirkt. Wenn Meltzer in der Analyse einen Traum hört, geht er nicht sofort auf Deutung oder Verständnis aus, sondern schiebt es im Gegenteil möglichst hinaus. In dieser Phase der Exploration assoziieren beide Beteiligten spielerisch, ohne Absicht, um den Traum herum. Das ist ein im Kern ästhetischer Prozess. Meltzer bringt seine Technik auf den Punkt in dem Satz: "While listening to your dream I had a dream” (Meltzer, 1984 S. 107). Den daran anschliessenden Prozess der Deutung beschreibt er als das Einbringen einer Ernte, die Gestaltung dessen, was in der Exploration sich zu entfalten begonnen hatte.

Der Unterschied zwischen den beiden Ansätzen ist, dass Meltzers Verständnis der reinszenierten archaischen Interaktion deutlich auf das postkleinianische Repertoire von Einverleibung und Ausstossung, Verfolgung und Neid verweist, während Morgenthaler, der einen ichpsychologischen Ansatz vertritt, allerdings durch eine kritische, dialektische Haltung sozusagen entmechanisiert, wesentlich offener ist für die Vielfalt der Traumfigurationen. Er betrachtet den Traum als dramaturgisch organisiertes Geschehen, als Traumtheater, das sein Publikum, den Träumer, am Aufwachen hindern soll.
Im Schlaf sitzt der Träumer als Besucher in einem Theater. Der Vorhang geht auf, und er sieht auf der Bühne eine Szene, zum Beispiel den Sommernachtstraum von Shakespeare. Hinter den Kulissen sitzen die Traumregisseure. Das sind die Instanzen der unbewussten Ichanteile, die dafür sorgen, dass auf der Bühne alles so vor sich geht, wie es geplant ist (...). Die Theaterdirektion wählt Stücke, die beim Publikum Erfolg haben sollen. Sie berücksichtigt bei ihrer Wahl das Traditionelle, die Erinnerung und die derzeitige Stimmung im Volk. Das ist die emotionale Bewegung im Unbewussten. Das Volk aber ist unzufrieden, weil im Theater nie das aufgeführt wird, was es wirklich will. Unzufrieden sind vor allem die ungesitteten, schwer unter Kontrolle zu haltenden Aufbegehrer, die alles immer in Unordnung bringen wollen. Während der Theateraufführung drängen diese Leute von der Strasse durch den Artisteneingang ins Theater. Einige sind betrunken, andere kommen mit einem Hund oder einem Ziegenbock. Eine schreiende Frau ist auch dabei und vieles mehr. Diese Leute sind Störfaktoren und drohen auf die Bühne durchzubrechen und Shakespeares Sommernachtstraum in eine wilde Demonstration umzufunktionieren. Die Eindringlinge sind die unbewussten Triebregungen. Auf der Bühne muss alles schön und geregelt ablaufen, damit der Träumer, der im Theater sitzt, nicht erwacht. Das ist so, weil der Traum die Funktion hat, den Schlaf zu schützen. Nun kommen die Traumregisseure in Aktion. Ihnen steht das Arsenal der Vergangenheit von allem Erlebten zur Verfügung, um daraus die Requisiten und Verkleidungsmöglichkeiten zu wählen, mit denen sie die Eindringlinge so verändern, verkleiden und in Symbolkisten einschliessen, dass sie dann, wenn sie auf der Bühne erscheinen oder in den gewählten Requisiten auf die Bühne geschoben werden, die Szene, die gerade gespielt wird, nicht stören. Je intensiver und drängender die Impulse sind, die zur Bühne gelangen wollen, je schneller das vor sich geht und je grösser die Menge der Eindringlinge ist, desto schwieriger wird es für die Traumregisseure, alles rechtzeitig zuzudecken. Es kann dann vorkommen, dass eine Prinzessin auf der Bühne noch irgendwo ein Horn trägt, weil es der Traumregie nicht mehr gelungen ist, den Ziegenbock ganz zu verkleiden. Der Träumer, der im Publikumsraum sitzt, sagt 
sich dann: "Merkwürdig, was in diesem Stück alles vorkommt».

(Morgenthaler, 1984, S. 81 ff.)

Selbst wenn Morgenthalers Theatermodell des Traums zunächst eine Veranschaulichung des innerpsychischen Traum-Synthetisierungsprozesses darstellt, insoweit also der Ichpsychologie zuzuordnen wäre, enthält sie gerade in ihrer uneigentlichen, metaphorischen Bedeutungsebene erhebliches Potential für eine interpersonale Theorie. Indem sie die Traumverfertigung auf einer inneren Bühne verortet, auf der einem konfliktscheuen bürgerlichen Publikum Beruhigendes vorgeführt werden soll, das jedoch durch eindringende abjekte Gestalten in Gefahr gebracht wird, entfaltet sie eine implizite Sozialtheorie des Traums. Erst in der sozialen Matrix wird ja das Nichtsymbolisierte als beunruhigend und gefährlich gesehen; diese soziale Matrix, in Morgenthalers Metapher das bürgerliche Theater, wird sowohl in der primären Traumsozialisation konstituiert als auch in der therapeutischen Interaktion reinszeniert. Wenn die Eltern dem Kind sagten, «Träume sind Schäume» und damit das präsymbolische, eigensinnige Begehren aus der kommunikativ geregelten Wirklichkeit ausschliessen, ist auch die analytische Stunde ungeachtet aller guten Vorsätze ein Schauplatz, auf dem das Unerhörte gemeinsam vom Bewusstwerden abgehalten wird. Erst die Analyse dieser Abwehr macht es erkennbar. Die Traumerzählung enthält einen dialektischen Widerspruch - Morgenthaler (1978) hat ja den Begriff der Dialektik auch für seine behandlungstechnische Theorie in Anspruch genommen. Sie versuch das in der bewussten Interaktion, auch der Analyse, Unsagbare, Ausgeschlossene, in den Raum eindringen zu lassen und es gleichzeitig zu verbergen. Die unbewusste Ichleistung ist seine Maskierung im Traumsymbol, die Tendenz aber, auf die es Morgenthaler ankommt, ist die der Beunruhigung. Die Traummitteilung ist ein dynamisches Geschehen, das die gesicherte Beziehung in Frage stellt und den gewohnten Diskurs auszuhebeln versucht, sich zugleich aber gerade auf den sicheren Raum der Beziehung verlässt. Morgenthalers Theatermetapher hebt die eminente Bedeutung der zeitlichen Struktur des Traumablaufs hervor - zu den traumhaft-merkwürdigen Bildern (die Prinzessin mit dem Horn) kommt es dann, wenn die Abwehr mit dem Drängen des Triebes nicht Schritt halten kann. Ähnlich in der Szene der Traummitteilung: Auch hier werden die unordentlichtriebhaften Wunschelemente von den Ordnungshütern des Narrativs nur mühsam bewältigt, es entsteht eine aufgeladene Stimmung im Raum. Die Traumerzählung als dynamisches Narrativ folgt den Gesetzen des Erzählens, die sie durch ihre Überdeterminiertheit zugleich unterläuft. In sozialwissenschaftlicher Sicht dient das Narrativ der Herstellung von Identität und Geschichtlichkeit (Gergen, 1994). Innere und äussere Erzählungen sind dramaturgisch konstruiert und dienen dazu, den Adressaten in die Erzählsituation einzubinden (Boothe, 2011; Bruner, 1991). Um Aufmerksamkeit auf sich zu ziehen - und das gilt auch für innere Aufmerksamkeit-, muss die Erzählung von etwas Unerwartetem, Aussergewöhnlichen handeln. Tatsächlich ist dieses Umgehen mit dem Ungewöhnlichen vermutlich der Ursprung des Träumens (Moser \& von Zeppelin, 1996). In diesen Erzählungen wird das Selbst fortwährend und je nach aktuellem Bedarf produziert (Gergen \& Gergen, 1986; Gergen, 1994). Akte des autobiographischen Erinnerns treten nur in sozialen Situationen auf und zwar in Form von Erzählungen, die kulturspezifischen und historisch variablen Regeln unterliegen. Sie sind auf ein Ziel, eine Idee oder eine abschliessende Pointe hin orientiert, wählen die zu diesem Endpunkt passenden Ereignisse aus, sind zeitlich geordnet und kausal verknüpft und werden durch Abgrenzungszeichen als autobiographische Erinnerung ausgewiesen. Die soziale Situation, in der sie auftreten, ist «ein kulturell choreographierter Tanz (...), ein gelebtes Narrativ» (Gergen, 1994, S. 91 ff., S. 97).

\section{$5 \quad$ Ein Beispiel}

Die beziehungsanalytische Traumdeutung, deren Grundlagen und technische Annahmen bisher besprochen wurden, wurde in früheren Veröffentlichungen an Beispielen gezeigt - am unbewussten Dialog zwischen Träumer und Analytikerin in einer Studie zu Traumnarrativ und Gedächtnis (Hamburger, 1998b), in der Rekonstruktion von Timing und Spannungsdramaturgie der Traumerzählung (Hamburger, 2006a) und in einer Untersuchung zu Gegenübertragungsträumen (Hamburger, 2010). Das vorliegende Heft kreist in zahlreichen Beiträgen um das Werk von Fritz Morgenthaler - deshalb möchte ich die beziehungsanalytische Traumdeutung hier an einer Reanalyse eines Traumbeispiels von Morgenthaler verdeutlichen

Eine Patientin erzählte Morgenthaler den folgenden Traum ihres Kindes, zusammen mit der Vorgeschichte:

Das Kind hilft den Eltern Kirschen pflücken und muss die Früchte in den Korb legen und nach Hause tragen. Es ist gut erzogen und folgsam. Deshalb hat es alle Kirschen nach Hause gebracht und keine unerlaubt gegessen. Das Kind geht schlafen und träumt, es habe einen Korb voller Kirschen vor sich und esse sie alle auf. (Morgenthaler, 1984, S. 70) 
Prima vista ist dies ein Kindertraum nach dem von Freud definierten Muster der «einfachen Wunscherfüllung», für die er in der «Traumdeutung» (1900 S. $132 \mathrm{ff}$.) Beispiele anführt: Seine Tochter Anna, die im Alter von einem Jahr und sieben Monaten von «Eierpeis, Papp» träumte, sein Neffe Hermann (ein Jahr und zehn Monate), der - eine besonders starke Parallele zu Morgenthalers Beispiel «alle Kirschen aufgessen» hat. Aber Morgenthaler (1984, S. 70) lässt es bei dieser Klassifizierung nicht bewenden, er geht weiter: Nach seiner Auffassung ist die Wunscherfüllung eine unbewusste Ichleistung, nicht aber die «unbewusste Tendenz» des Traums, die in der Szene der Mitteilung anzusiedeln ist. Um diese zu eruieren, fragt er seine Patientin: «Woher wissen Sie, dass das Kind diesen Traum gehabt hat?» Die Analysandin antwortet: «Das Kind hat den Traum unserem Dienstmädchen erzählt.» Im weiteren Gespräch erfährt der Analytiker, dass das Dienstmädchen den Traum der Mutter berichtet hat und die Mutter ihn jetzt weitererzählt. Morgenthaler schliesst:

Daraus ergibt sich, dass das Kind nicht zur Mama gegangen ist um ihr den Traum zu erzählen, sondern das Dienstmädchen gewählt hat, wohl wissend, dass dieses Dienstmädchen den Traum der Mutter weitererzählt. (...) Richte ich meine Aufmerksamkeit darauf, wie die Mutter den Traum ihres Kindes dazu benutzt, vorzuführen, wie frei ihr Kind träumen könne, dabei aber die Kolportage des Traumes über das Dienstmädchen benötigt, um mit dem Unbewussten ihres Kindes in Kontakt zu treten, wird der Konflikt, in dem sich das Kind befindet, spürbar. Da die Frau, die mir den Traum erzählt hat, bei mir in Analyse stand, verfügte ich über zahlreiche Informationen, die sich bei mir zu der Vorstellung verdichtet haben, dass dieses Kind Angst hat, so manipuliert zu werden, wie es die Kirschen manipuliert. Es hat Angst, von der Mama gefressen zu werden, wie es selbst die Kirschen isst. Das will das Kind vermeiden, indem es den Traum dem Dienstmädchen erzählt, so wie der Hänsel im Märchen der blinden Hexe statt seines Fingers ein Knöchelchen hinstreckt, um sie in den Glauben zu lassen, er sei noch viel zu mager, um schon verspeist zu werden. (Morgenthaler, 1984, S. 71)

Diese Wendung illustriert eindrücklich die Besonderheit von Morgenthalers szenischem Verständnis des Traums. Die Patientin erzählt ihrem Analytiker den
Traum ihrer Tochter (so schreibe ich aus der Erinnerung; tatsächlich bleibt es in Morgenthalers Text offen, ob das Kind ein Sohn oder eine Tochter war) in der Annahme, eine leicht zu entschlüsselnde Botschaft zu überbringen. Aber warum stellt sie ihren Analytiker vor diese «leichte» Aufgabe? Was will sie ihm unbewusst mitteilen, wenn sie ihn - einen berühmten Traumspezialisten - mit solchem Kinderkram beschäftigt? Morgenthalers Frage, woher sie von dem Traum wisse, ist keineswegs selbstverständlich. Sie könnte sogar ablenkend wirken, als ob dies etwas Nebensächliches sei. Sie ist aktiv, und es liesse sich einwenden, dass sie die freie Assoziation der Analysandin beschränke. Aber sie entspringt einem Verdacht, der aus der Teilhabe an der Szene stammt - der Analytiker kann sich des Eindrucks nicht erwehren, dass diese vordergründig harmlose Erzählung einen doppelten Boden habe. Deshalb fragt er spontan nach - und wie sich zeigen wird, sehr kreativ. Die Antwort lässt ihn aufhorchen: vom Dienstmädchen. Dieses folgsame, brave Kind, vorgeführt als Traum-Musterkind, welches sogar Freudsche Träume reproduziert, jubelt der Mutter via Dienstmädchen einen Traum unter, zur Weitergabe an den Analytiker. Hier ist der Punkt, an dem Morgenthaler die unbewusste Tendenz des Traums festmacht. Dieser Traum soll ihm sagen: Ich bin ein braves Kind, aber in all meiner Folgsamkeit stosse ich doch den Analytiker mit der Nase darauf, dass hier etwas nicht stimmt. Ich erzähle den Traum dem Dienstmädchen. Der Mama erzähle ich ihn nicht. So kommt Morgenthaler zu seiner Deutung, die freilich trotzdem noch etwas befremdlich klingt: das Kind fürchtet, von der Mutter gefressen zu werden. Morgenthaler belässt es bei der Andeutung, er habe aus der Analyse der Mutter zahlreiche Informationen, die ihn zu dieser Annahme bringen. Vielleicht wäre sein Schluss leichter nachvollziehbar, wenn er an dieser Stelle mehr über die eigentliche Szene der Traummitteilung an ihn selbst mitgeteilt hätte - und hier würde eine beziehungsanalytische Arbeit mit dem Traum deutlicher ansetzen wollen. Die Szene, das Hier und Jetzt, ist ja eine Mutter, die ihrem Analytiker den Traum ihres Kindes erzählt. Es wäre naheliegend, sich auf die unbewussten Implikationen dieser Mitteilungsszene zu konzentrieren, anstelle einer Fern-Analyse des Kindes. Die Analysandin präsentiert dem für seine Traumseminare bekannten Analytiker einen Vorzeigetraum von einem mustergültig folgsamen Kind. Sie will ihn damit vielleicht testen und ihm beweisen, dass sie ein völlig gesundes Kind hat. Aber ihr Unbewusstes setzt den «Fehler» durch, ihn durch die Anhäufung dieser Grossartigkeiten misstrauisch zu machen. Er fragt nach, und es kommt heraus, dass dieses Kind den Traum nur indirekt erzählt und das bringt den Analytiker auf die Fantasie, es könnte Angst vor seiner Mutter haben. Bedeutsam ist, dass diese lediglich eine Fantasie ist, denn sie muss für 
das Kind nicht zutreffen. Für die Mutter aber, die Analysandin, könnte es lesbar sein als szenische Mitteilung: «Ich möchte zeigen, was für ein gesundes Kind ich habe, aber eigentlich fürchte ich, dass mein Kind Angst vor mir hat und deswegen so fügsam ist.» Und in einer zweiten Ebene: «Ich bin selbst ebenso fügsam und füttere Sie mit leicht verdaulichen Träumen, aber eigentlich richte ich dabei ein ziemliches Durcheinander an und versuche, Sie auf den Leim zu führen.» Erst die szenische Ebene der Traummitteilung (aus der auch meine Fehlerinnerung herrührt, es handle sich bei dem träumenden Kind um eine Tochter) lässt uns diese möglichen Kernbotschaften des Traumes verstehen.

\section{Zusammenfassung und Ausblick auf weitere Forschung}

Die Mitteilungsszene des Traums in der Analyse ist hoch verdichtet. Indem der Analysand die Textsorte «Traumerzählung» wählt, um mit dem Analytiker zu kommunizieren, bietet er eine Rede an, die er als Kind als Rede von der exklusiven inneren Welt entdeckt hat, welche zudem eine Welt ist, die er selbst nicht versteht. Das Spezifische der introspektiven Traumerfahrung ist ja, dass Träume in den seltensten Fällen so einfach sind wie der Kirschentraum - meist verwundern sie in Form und Inhalt den Träumer selbst. An dieser inneren Wunderwelt lässt der Analysand den Analytiker durch seine Rede teilhaben. Indem er sie narrativ vermittelt, evoziert er zugleich im Rezipienten affektive Teilnahme, und steuert sie durch die Gestaltung der temporalen Dramaturgie der Traumerzählung (Hamburger, 2006a).

Hier eröffnet sich ein weites und aktuelles Forschungsfeld, auf dem die dichte Erfahrung des psychoanalytischen Prozesses mit Untersuchungsergebnissen und -methoden aus anderen Wissenschaften in Beziehung gesetzt werden kann. In der Konversationsanalyse werden Kontexte des gesprochenen Worts untersucht und die genaue zeitliche Abstimmung der Wechselrede in der gemeinsamen Erzeugung von Sinn (Buchholz, 2013, 2014; Buchholz \& Gödde, 2013). Die SzenischNarrative Mikroanalyse (Hamburger, 2015, 2017 im Druck) untersucht auf einer psychoanalytischen Grundlage die szenische Bewegung in Gesprächssituationen, um signifikante Momente der Begegnung im Sinne von Stern (2004) zu identifizieren. Interdisziplinäre Forschungsgruppen arbeiten daran, den von der Boston Change Process Study Group (2002) ausgerufenen hervorgehobenen "Moment of Meeting” empirisch reichhaltig zu beschreiben (Breyer, Buchholz, Hamburger \& Pfänder, 2017, im Druck sowie Brandstetter, Buchholz, Hamburger, Reich \& Wulf, 2017, in Vorbereitung). Beiträge kommen aus der lexikalischen Analyse nach Mergenthaler (Bleimling, 2016), der Motion Energy Analysis nach Ramseyer (ebd.), der Musiktherapie (Hamburger \& Metzner, 2017 im Druck), der Tanz- und Bewegungswissenschaft (Heller, 2017, im Druck), der Linguistik (Buchholz \& Reich, 2015) und anderen.

Traumerzählungen als hervorgehobene Momente therapeutischer Dialoge (Hamburger, 2006a) eignen sich für eine solche multidisziplinäre Analyse; die Durchführung steht noch als Forschungsdesiderat aus. Sie könnte empirisch untermauern, was die analytische Erfahrung bereits erbracht hat: Die Traumerzählung in der Analyse bietet eine privilegierte Einladung zum Dialog über eine Innenwelt und dieser Dialog wird von beiden Beteiligten auf multimodaler Ebene verhandelt.

\section{Literatur}

Alt, P.-A. (2002). Der Schlafder Vernunft. Literatur und Traum in der Kulturgeschichte der Neuzeit. München: C. H. Beck.

Altmeyer, M. (2001). Das Ende der Amöbensage lässt auf sich warten. Primärer Narzissmus oder primäre Intersubjektivität - bloss erkenntnistheoretisch ein Widerspruch. Psyche - Zeitschrift für Psychoanalyse und ihre Anwendungen 55, 619-624.

Aron, L. (1989). Dreams, Narrative and the Psychoanalytic Method. Contemporary Psychoanalysis 25 (1), 108-127.

Ávila, A., \& Domínguez, R. (2014). Dreams and Change Processes in an Ex-prostitute Under Relational Psychoanalytic Psychotherapy: Ferenczian and Relational Views. International Forum of Psychoanalysis 23, 23-29.

Bauriedl, T. (1980). Beziehungsanalyse. Frankfurt/M.: Suhrkamp.

Benjamin, J. (2009). A Relational Psychoanalysis Perspective on the Necessity of Acknowledging Failure in Order to Restore the Facilitating and Containing Features of the Intersubjective Relationship (the Shared Third). The International Journal of Psychoanalysis 90, 441-450.

Binswanger, R. (2016). Dream Diagnostics. Fritz Morgenthaler's Work on Dreams. The Psychoanalytic Quarterly 85 (3), 727-757.

Binswanger, R. (1986). Zur Traumtechnik Fritz Morgenthalers im Traumseminar und in der psychoanalytischen Praxis. In: Morgenthaler, F: Der Traum. Fragmente zur Theorie und Technik der Traumdeutung (S. 177-186). Frankfurt/M: Qumran.

Boothe, B. (2010). Das Narrativ. Biografisches Erzählen im psychotherapeutischen Prozess. Stuttgart: Schattauer. 
Boston Change Process Study Group (2002). Das Implizite erklären: die lokale Ebene und der Mikroprozess derVeränderung in der analytischen Situation. Psyche - Zeitschrift für Psychoanalyse und ihre Anwendungen 58, 935-952. Brandstetter, G., Buchholz, M., Hamburger, A., Reich, U. \& Wulf, C.(Hrsg.) (2017, in Vorbereitung). Rhythmus Balance Resonanz - Multiperpektivische Analysen. Paragrana.

Brazelton, T. B. \& Cramer, B. G. (1989). Die frühe Bindung. Die erste Beziehung zwischen dem Baby und seinen Eltern. Stuttgart: Klett-Cotta 1991.

Breyer, T., Buchholz, M, Hamburger, A. \& Pfänder, S. (Hrsg.) (2017, im Druck). Resonanzen in Interaktion - theoretische Fundierung und praktische Relevanz in Therapie, Alltag und Kunst. Bielefeld: Transcript.

Bruner, J. S. (1991). Self-Making and World-Making. Wie das Selbst und seine Welt autobiographisch hergestellt werden. Journal für Psychologie 7 / 1999 (1), $11-21$.

Buchholz, M. B. (2014). Die Feinheiten therapeutischen Sprechens. Konversationsanalyse eines psychoanalytischen Erstgesprächs. In I. Bozetti, I. Focke, I. Hahn (Hrsg.), Unerhört - Vom Hören und Verstehen. Die Wiederentdeckung der grundlegenden Methoden der Psychoanalyse (S. 219240). Stuttgart: Klett-Cotta.

Buchholz, M. B. (2013). Mikroprozesse therapeutischer Interaktion studieren! Folgerungen aus Outcome- und Prozessforschung für die professionelle Praxis der Psychoanalyse. In B. Boothe, P. Schneider (Hrsg.) Die Psychoanalyse und ihre Bildung (S. 85-120). Zürich: Sphères (Reihe Sphèreessays).

Buchholz, M. B. \& Reich, U. (2015). Dancing Insight. How a Psychotherapist uses Change of Positioning in Order to Complement Split-Off Areas of Experience. Chaos and Complexity Letters 8, 121-146.

Buchholz, M.B., Gödde, G. (2013). Balance, Rhythmus, Resonanz: Auf dem Weg zu einer Komplementarität zwischen «vertikaler» und «resonanter» Dimension des Unbewussten. Psyche - Zeitschrift für Psychoanalyse und ihre Anwendungen 67, 844-880.

Caligor, L. (1996). The Clinical Use of the Dream in Interpersonal Psychoanalysis. A Dream Specimen. Psychoanalytic Dialogues 6, 793-811.

Conci, M. (2011). Sullivan und die intersubjektive Sichtweise. Forum der Psychoanalyse 27 (2), 139-150.
Conci, M. (2005). Sullivan neu entdecken. Leben und Werk Harry Stack Sullivans und seine Bedeutung für Psychiatrie, Psychotherapie und Psychoanalyse. Giessen: Psychosozial.

Derrida, J. (1967). Die Schrift und die Differenz. dt. Frankfurt/M: Suhrkamp 1972.

Deutsch, H. (1926). Okkulte Vorgänge während der Psychoanalyse. Imago 12, 418-433.

Dornes, M. (1993). Der kompetente Säugling. Die präverbale Entwicklung des Menschen. Frankfurt/M.: Fischer, 13

Erdheim, M. (1984). Die gesellschaftliche Produktion von Unbewusstheit. Eine Einführung in den ethnopsychoanalytischen Prozess. Frankfurt/M.: Suhrkamp.

Fosshage, J.L. (2013a). Dream Interpretation Revisited. In A. Goldberg (ed.), Frontiers in Self Psychology, Progress in Self Psychology, 3 (S. 161-175).

Fosshage, J.L. (2013b). The Dream Narrative: Unconscious Organizing Activity in Context. Contemporary Psychoanalysis 49 (2), 253-258.

Freud, S. (1950). Aus den Anfängen der Psychoanalyse. Briefe an Wilhelm Fliess, Abhandlungen und Notizen aus den Jahren 1887- 1902. Frankfurt/M.: Fischer, 2. korr. Aufl. 1975.

Freud, S. (1905). Bruchstück einer Hysterie-Analyse. GWV.

Freud, S. (1900). Die Traumdeutung. G.W. I/II.

Freud, S. \& Breuer, J.(1895). Studien über Hysterie. Leipzig und Wien: Deuticke. (wiederabgedruckt ohne Breuers Beiträge in GW I, 77-312).

Gergen, K. J. (1994). Mind, Text, and Society: Self-memory in Social Context. In U. Neisser \& R. Fivush (eds), The Remembering Self (pp. 78-104). Cambridge: Cambridge University Press (=Emory Symposia in Cognition 6).

Gergen, K.J. \& Gergen, M.M. (1986). Narrative Form and the Construction of Psychological Science. In T. R. Sarbin (Ed.), Narrative Psychology. The Storied Nature of Human Conduct (pp. 22-43). NewYork, Westport, London: Praeger.

Greenberg, J. R. \& Mitchell, S. A. (1983). Object Relations in Psychoanalytic Theory. Cambridge/London: Harvard University Press.

Hamburger, A. (2016). Psychoanalyse und Philosophie. In H. Hierdeis (Hrsg.), Austauschprozesse: Psychoanalyse und andere Humanwissenschaften (S. 64-88). Göttingen: Vandenhoeck \& Ruprecht.

Hamburger, A. (2013). Via Regia und zurück. Traumerzählungen und ihre Resonanz. In B. Janta, B. Unruh \& S. Walz-Pawlita (Hrsg.), Der Traum (S. 123-143) Giessen: Psychosozial-Verlag. 
Hamburger, A. (2010). Traumspiegel. Gegenübertragungsträume in der Beziehungsanalyse. In H. Hierdeis (Hrsg.), Der Gegenübertragungstraum in der psychoanalytischen Theorie und Praxis (S. 23-50). Göttingen: Vandenhoeck \& Ruprecht.

Hamburger, A. (2006a). Traum und Zeit. Traumerzählungen als Elemente der Spannungsdramaturgie. Forum der Psychoanalyse 22, 23-43

Hamburger, A. (2006b). Der Kindertraum als Paradigma der Traumauffassung. Analytische Kinder- und Jugendlichenpsychotherapie 37 (3), 321-363.

Hamburger, A. (2000). Traumerzählung und interaktives Gedächtnis. Zur Psychoanalyse der Identität. In M. Neumann (Hrsg.), Erzählte Identitäten. Ein interdisziplinäres Symposion (S. 253-279). München: Fink.

Hamburger, A. (1999). Der Kindertraum - Einfache Wunscherfüllung, Selbstnarrativ Mitteilung? In H. Bareuther, K. Brede, M. Ebert-Saleh, K. Grünberg (Hrsg.), Traum, Affekt und Selbst. Frankfurt/M.: edition diskord 1999 (= Psychoanalytische Beiträge Bd. 1).

Hamburger, A. (1998a). Narrativ und Gedächtnis. In M. Koukkou, M. LeuzingerBohleber \&W. Mertens (Hrsg.), Erinnerung von Wirklichkeiten. Psychoanalyse und Neurowissenschaften im Dialog. Bd. 1: Bestandsaufnahme (S. 223-286) Stuttgart: Verlag Internationale Psychoanalyse.

Hamburger, A. (1998b). Solo mit Dame. Traumgeschichten einer Psychoanalyse. In M. Leuzinger-Bohleber, W. Mertens \& Martha Koukkou (Hrsg.), Erinnerung von Wirklichkeiten. Psychoanalyse und Neurowissenschaften im Dialog. Bd. 2: Folgerungen für die psychoanalytische Praxis (96-127). Stuttgart:Verlag Internationale Psychoanalyse.

Hamburger, A. (1998c). Des Rätsels Lösung. Rückblick auf die ursprüngliche Szene der Traumerzählung. In Traumwerkstatt (Hrsg.), Träume in der Paartherapie Thea Bauriedl, Eva Jaeggi und Helm Stierlin im Gespräch über einen Paartraum. Göttingen: Vandenhoeck und Ruprecht.

Hamburger, A. (1995) Entwicklung der Sprache. Stuttgart: Kohlhammer.

Hamburger, A. (1987). Der Kindertraum und die Psychoanalyse. Ein Beitrag zur Metapsychologie des Traums. Regensburg: Roderer.

Haubl, R. \& Mertens, W. (1996). Der Psychoanalytiker als Detektiv. Eine Einführung in die psychoanalytische Erkenntnistheorie. Stuttgart: Kohlhammer.

Heinrichs, H.-J. (1986). Vorbemerkung. In F. Morgenthaler, Der Traum. Fragmente zur Theorie und Technik der Traumdeutung (S. 11-16). Frankfurt/M: Edition Qumran im Campus-Verlag, Neuauflage Giessen: Psychosozial (2004).

Honneth, A. (1992). Kampf um Anerkennung. Frankfurt/M. (Suhrkamp).
Lorenzer, A. (1985). Der Analytiker als Detektiv, der Detektiv als Analytiker. PsycheZeitschrift für Psychoanalyse und ihre Anwendungen 39 (1), 1-11.

Lorenzer, A. (1970). Sprachzerstörung und Rekonstruktion. Frankfurt/M: Suhrkamp Meltzer, D. (1984). dt.: Traumleben. Eine Überprüfung der psychoanalytischen Theorie und Technik. München, Wien:Verlag Internationale Psychoanalyse 1988.

Mitchell, S.A. (2000). Bindung und Beziehung. Auf dem Weg zu einer relationalen Psychoanalyse. Giessen: Psychosozial.

Mitchell, S. A. (1997). Psychoanalyse als Dialog: Einfluss und Autonomie in der analytischen Beziehung. Giessen: Psychosozial, 2005.

Morgenthaler, F. (1986). Ein Traum als Beweismittel. In F. Morgenthaler, Der Traum (S. 17-52). Frankfurt a. M., Campus 1990.

Morgenthaler, F. (1984). Traumdiagnostik. Zur Bedeutung der formalen und strukturellen Gesichtspunkte. In F. Morgenthaler, Der Traum (S. 53-88). Frankfurt a. M., Campus 1990.

Morgenthaler, F. (1978). Technik. Zur Dialektik der psychoanalytischen Praxis Frankfurt/M.: Syndikat, Neuauflage Giessen: Psychosozial (2005).

Moser, U. \& von Zeppelin, I. (1996). Der geträumte Traum. Wie Träume entstehen und sich verändern. Stuttgart: Kohlhammer.

Nunberg, H. \& Federn, E. (Hg.) (1977) Protokolle der Wiener Psychoanalytischen Vereinigung Bd. 2. Frankfurt/M.: Fischer

Orange, D., Atwood, G. E. \& Stolorow, R.D. (1997).Intersubjektivität in der Psychoanalyse. Kontextualismus in der psychoanalytischen Praxis. Frankfurt/M.: Brandes \& Absel, 2001.

Raguse, H. (1998). Psychoanalytische Hermeneutik - Weltanschauung oder Regelcorpus?. Psyche - Zeitschrift für Psychoanalyse und ihre Anwendungen 52 (7), 648-703.

Reiche, R. (1999). Subjekt, Patient, Aussenwelt. Psyche-Zeitschrift für Psychoanalyse und ihre Anwendungen 53, 572-596

Solms, M. (1997). The Neuropsychology of Dreams. A Clinico-Anatomical Study. Mahwah NJ (Lawrence Erlbaum).

Spence, D. P.(1976). Clinical Interpretation. Psychoanalysis and Contemporary Science 5, 367-388

Stern, S. (2004). The Yin and Yang of Intersubjectivity. Progress in Self Psychology 20 3-20. 
Stern, D. N. (1989). Crib Monologues from a Psychoanalytic Perspective. In K. Nelson (ed.): Narratives from the Crib (pp. 309-319). Cambridge, London: Harvard University Press.

Stern, D. N. (1985). Die Lebenserfahrung des Säuglings. Stuttgart: Klett-Cotta 1992. Wachtel, P. L. (1976). Structure or Transaction?. Psychoanalysis and Contemporary Science 5, 101-136

\section{Angaben zum Autor}

Andreas Hamburger, Professor für Klinische Psychologie an der International Psychoanalytic University, Berlin. Forschungsschwerpunkte: Traum- und Sprachentwicklung, Soziales Trauma, Szenisch-narrative Mikroanalyse, Literatur- und Filmpsychoanalyse, Supervisionsforschung. Letzte Buchveröffentlichungen: Frauen- und Männerbilder im Kino. Genderkonstruktionen in La Belle et la Bête von Jean Cocteau. Giessen: Psychosozial 2015; W. Mertens \& A. Hamburger (Hrsg): Supervision - Konzepte und Anwendungen (2 Bände). Stuttgart: Kohlhammer 2016. 